\title{
DEPRESSIVE DISORDERS IN PATIENTS WITH MITOCHONDRIAL PATHOLOGY (MECHANISMS OF FORMATION, CLINICAL TYPOLOGY, SYSTEM OF CORRECTION AND PREVENTION)
}

\author{
Strelnikova I. \\ Kharkiv National Medical University, Ukraine \\ https://doi.org/10.35339/ic.7.1.49-56
}

\begin{abstract}
Summary
The article introduces a modern understanding of the functioning of mitochondria, the flow of the main biochemical processes that are related to energy metabolism. A generalized characteristic of the mitochondrial genome mutations and types of mitochondrial dysfunctions are presented. The modern ideas about the occurrence of mental disorders in patients with mitochondrial pathology are analyzed. Material and methods. In order to study depressive disorders, 171 patients of both sexes with mitochondrial pathology were examined, aged from 18 to 57 years. Results. The most common clinical and psychopathological features of the examined patients with MP were analyzed. The most common complication of MP was depressive state. An additional analysis of depressive disorders and their variants were carried out. The study involved screening of 26 families of patients with mitochondrial pathology, including 14 families of patients with MP, accompanied by depressive disorders and 12 families of patients with MP without affective disorders. High incidence of psychosomatic symptoms and depression were detected by mother's side relative probands. According to the results of the study, patients received pharmacotherapy and psychotherapy care, depending on the clinical manifestations of depressive symptoms and the degree of their severity. Psycho-educational work was conducted with patients and their families.

Keywords: antidepressants, anxiety, depression, mitochondrial diseases, mitochondrial dysfunction, psychoeducation, psychotherapeutic interventions.
\end{abstract}

\section{Introduction}

Mitochondrial diseases are caused by genetic and structural-biochemical defects of mitochondria, accompanied by a violation of tissue respiration and, as a consequence, a systemic defect in energy metabolism, which results in the most diverse combination of the most energydependent tissues and target organs: the brain, skeletal muscles and myocardium (mitochondrial encephalomyopathy), pancreas, organ of vision, kidneys, liver $[1,3,12]$.

Clinical disorders in the mentioned organs can develop at any age. At the same time heterogeneity of symptoms complicates the clinical diagnosis of these diseases $[2,4,9]$.

Corresponding Author:

Irina Strelnikova - MD, Associate professor of the Department of Psychiatrics, Narcology, Medical Psychology and Social Work of Kharkiv National Medical University. Kharkiv, Ukraine.E-mail: vodoley20001@ukr.net
The peculiarity of the functioning of mitochondria is the presence of its own mitochondrial genome, the circinate mitochondrial DNA (mtDNA), which contains 37 genes, whose products are involved in the process of energy generation in the respiratory chain of mitochondria $[1,2,11]$.

The mitochondria undergo major biochemical processes related to energy metabolism:

- a cycle of tricarboxylic acids (Krebs cycle),

- beta-oxidation of fatty acids;

- carnitine cycle;

- transportation of electrons in the respiratory chain;

- oxidative phosphorylation.

Any of the mentioned processes may be violated and cause mitochondrial failure. (Fig. 1).

The immediate cause of the emergence and development of mitochondrial dysfunction is the defect of the oxalic phosphorylation system, imperfection of the apparatus mechanisms, absence of histones, and presence of free oxygen radicals, by-products of aerobic respiration $[1,7,10]$. 


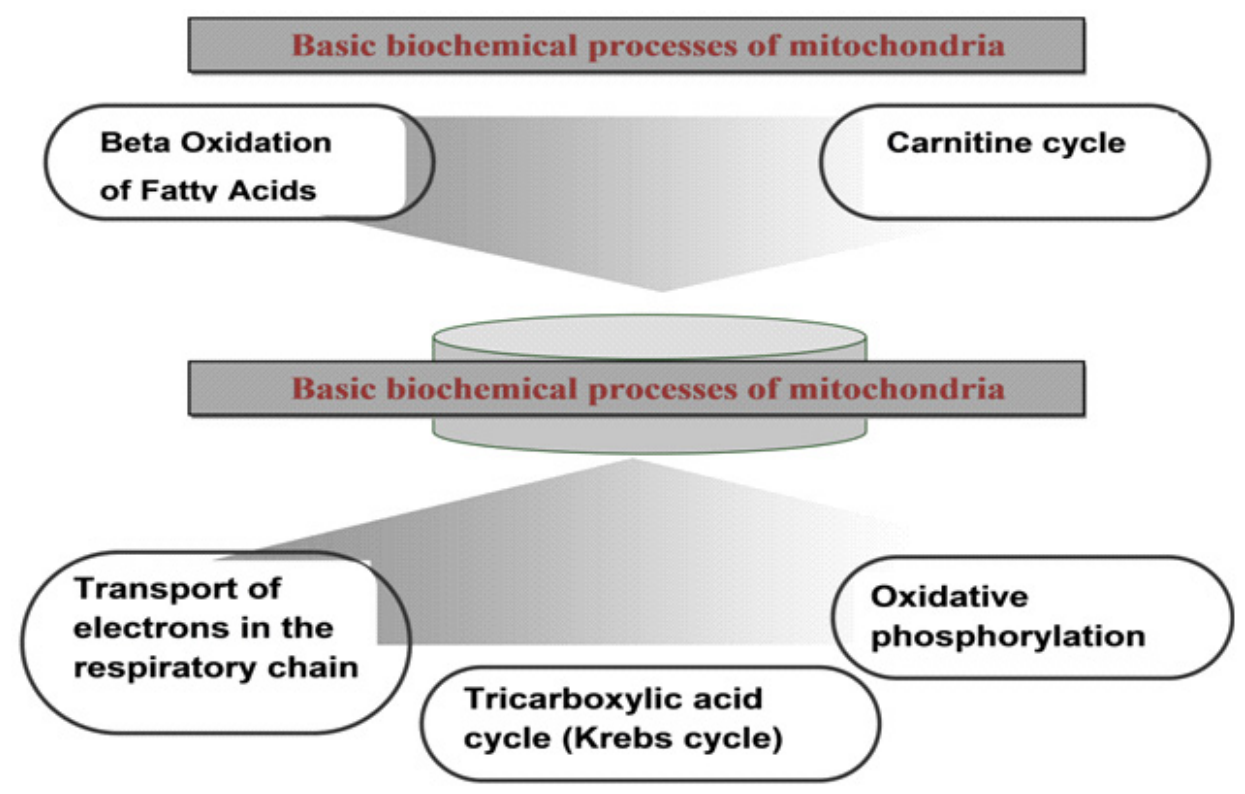

Fig. 1. Basic biochemical process of mitochondria

The main qualities of the mitochondrial genome are cytoplasmic imitation of genes, the lack of recombination (thus, the reorganization of the genetic material through the exchange of individual segments, DNA double helix areas) and the high rate of mutation occurrence.

The mitochondrial genome is different by:

1) Expressed instability

2) High speed of nucleotide substitutions

3) During the life of the individual, there are often somatic mutations.

For mutations in the mitochondrial genome, the phenomenon of heteroplasmia is characterized by the simultaneous presence of many copies of DNA (that is, mitochondria) that carry the normal or mutant allele and varies widely (1-99\%).

In the process of cell division, both types of DNA (with normal or mutant alleles) are distributed randomly between the daughter cells [12].

Therefore, in subsequent generations, one part of the cells can only have normal mtDNA, the second part is only mutant, and the third part is the normal and mutant type of mtDNA $[12,13]$.

The number of mitochondria with mutant mtDNA increases gradually. Due to the "lag of the period", future patients often reach puberty and give offspring that almost always carry the same mutations in mtDNA $[1,2]$.

When the amount of mutant copies of mtDNA reaches a cell of a certain concentration, the energy metabolism in the cells becomes significantly disturbed and manifests itself as a disease. (The peculiarity of hereditary mitochondrial diseases is often the complete absence of any pathological signs at the beginning of the patient's life) $[3,5]$.

Since mtDNA in the body has virtually exclusively maternal origin, during the transmission of the mitochondrial mutation, the offspring in the pedigree have a maternal type of imitation - all the "daughters" of the sick "mother" are ill $[8,10]$.

This is a typical pathological process that does not have etiological and nosological specificity.

It is manifested by pathobiochemical mechanisms of neurodegenerative disorders of a wide spectrum.

The development of mitochondrial dysfunction leads to:

- violation of reuptake of mediators (catecholamine, dopamine, serotonin);

- violation of ion transport, generation and conduction of the pulse, as well as the synthesis of de novo protein;

- violations of broadcasting and transcripts;

- "parasitic" energy-generating reactions that lead to the loss of energy stores of the nerve cell are activated.

Types of mitochondrial dysfunction:

Primary - as a consequence of an innate genetic defect.

Secondary - under the influence of various factors: hypoxia, ischemia, oxidative and nitrosating stress, the expression of proinflammatory cytokines.

Changes in the energy metabolism of the brain violate its work and lead to the development of mental disorders $[6,8]$.

From 40 to $60 \%$ of the energy of ATP in neurons is spent to support the ionic gradient on 
their outer envelope and the transfer of the nerve impulse. Violation of the function of the mitochondria leads to a violation of the processes of synaptic transmission of the nerve cell, a violation of cell growth and apoptosis.

Therefore, the violation of the function of the central nervous system in classical "mitochondrial diseases" is of great importance and makes it possible to call the main symptom "mitochondrial encephalomyopathy" $[9,14]$.

Clinically in the foreground there are such brain disorders as dementia, seizures and strokelike episodes.

The severity of these forms of the disease, combined with severe somatic disorders, can be so great that other violations associated with the person with emotional or personal changes, remain in the shadows $[7,11]$.

In recent years, the role of the contribution of mitochondrial disorders to the development of mental illness, foremost of the depressive disorder, is widely discussed. Indirect confirmation of the connection between mitochondrial and some mental illness is also a tendency to the focus of their clinical manifestations.

According to previous studies, $20 \%$ of patients with mitochondrial diseases had an associated BAR, with $0.38 \%$ of BAR having DNA polymerase mutations (y) (DNA polymerase gamma-POLG) that cause the development of mitochondrial diseases. In a new work (2018) conducted by Japanese scientists at the RIKEN Center for Brain Science (RIKEN), it has been established that mitochondrial dysfunction may affect the activity of serotonergic neurons with A1NT1 mutations [20].

\section{Purposes, subject and methods}

2.1. The purpose. Analyzing the abovementioned information, we conducted our own study in order to study the presence of depressive disorders in patients with mitochondrial pathology and develop a comprehensive approach to their therapy.

\subsection{Subjects and methods.}

Object of research: on the basis of Kharkiv Specialized Medical Genetic Center, 171 patients with mitochondrial pathology of both genders aged from 18 to 57 years were examined. Screening survey of 26 families of patients with mitochondrial pathology was conducted.

In this work a set of research methods was used: clinical and psychopathological, with the study of presentation, assessment of the mental status of patients, the allocation of the main psychopathological syndromes, their dynamics, as criteria of diagnosis selected criteria MKH-10, patients were further examined by a physician geneticist; Clinical-anamnestic, psychodiagnostic using the clinical Scales of Anxiety and Depression of Hamilton (M. Hamilton, 1967), adapted to MKH-10 (G.P. Pankeleva, 1988), Questionnaire of Neuro-Psychic Stress (T.A. Nemchin, 1984), SCL Scale, catamnestic and methods of mathematical statistics. The results are presented in the form of an average value \pm mistake of representativeness at a probability level of $\mathrm{p}<0.05$.

\section{Conflict of interests}

There is no conflict of interests.

\section{Results and discussion}

According to the results of the first stage of the study, all patients were divided into two groups. The first group consisted of patients with mitochondrial pathology without signs of mental disorders $(32.7 \%$ of the surveyed), the second group included $67.3 \%$ of patients with mitochondrial pathology, which had signs of depressive disorders (Fig. 2).

At the second stage, patients with mitochondrial pathology with signs of depression were analyzed in details. According to the results of the analysis,

\section{The 1st stage \\ - With no mental disorder \\ - Depressive disorders}

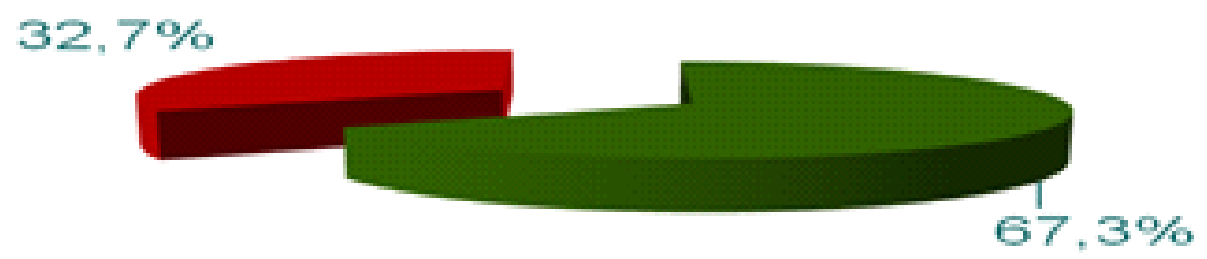

Fig. 2. Results of research of the 1st stage (\%) 
the patients were divided into three groups: the first group included patients with mild manifestations of depression (17.6\%), the second group included patients with moderate depression $(72.1 \%)$ and the third group were patients with severe manifestations of depression (10.3\%) (Fig. 3). pathology with mild to moderate depression, no significant differences between women and men were recorded, but both anxiety and depression rates are somewhat higher in the female group $(p \leq 0.001)$. In patients with mitochondrial pathology with mild to moderate depression, signs

\section{The 2nd stage}

\section{- Mild depressive manifestations \\ - Moderate depressive manifestations Severe depressive manifestations}

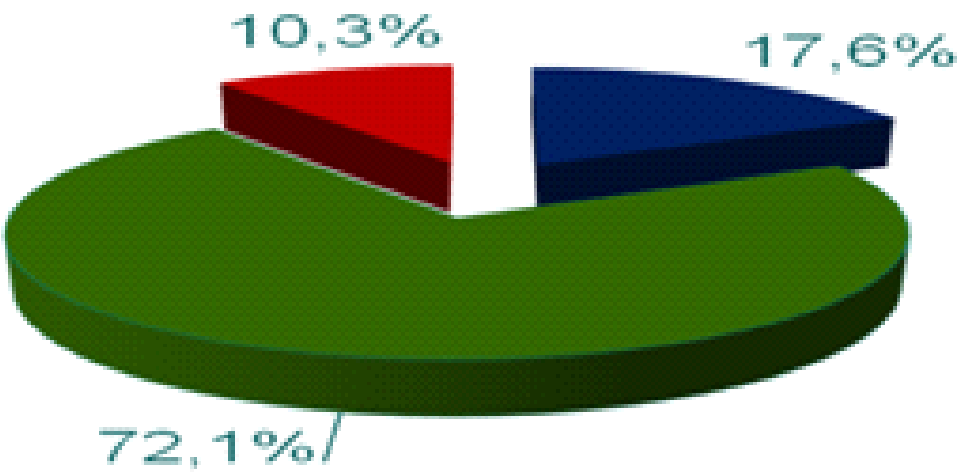

Fig. 3. Results of the second stage study $(\% ; p<0.05)$

In the study of the severity of anxiety and depressive manifestations in patients with mild, moderate and severe depression, the data presented in Figure 4 were obtained.

It is noteworthy that in patients with mitochondrial pathology of mild to moderate depressive manifestations, higher rates of both anxious and depressive symptoms were given to women $(p<0.05)$. In patients with mitochondrial of anxiety and depression were also higher among women $(\mathrm{p} \leq 0.001)$.

Analyzing the results of evaluation of the expressiveness of the nervous-psychic stress in the examined patients with mitochondrial pathology, we can notice that the level of pediatric neuro-psychic stress is significantly increased in men compared to women $(\mathrm{p}<0.05)$. The level of intense neuropsychiatric stresses in men and

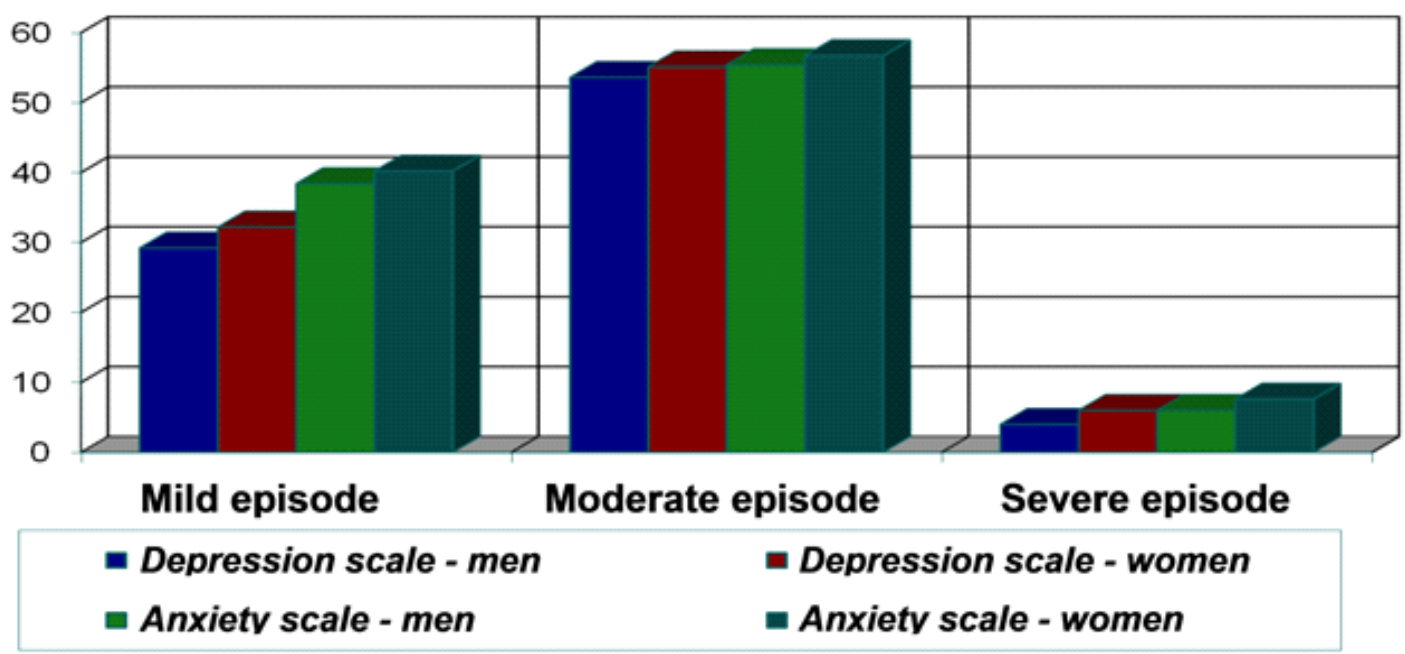

Fig. 4. Level of anxiodepressive manifestations in patients with mitochondrial pathology $(\mathrm{p}<0.05 ; \mathrm{p} \leq 0.001)$ 
women has approximately the same rates $(p \leq 0.001)$. On the contrary, assessing the level of extensive nervous and mental stress, there is a significant increase in this indicator in the female group $(\mathrm{p}<0.05)$. Fig. 5 .

Additional analysis of the variants of depressive disorders was carried out to distinguish anxietydepressive (50.3\%), asthenodepressive (18.5\%),
Asthenodepressive variant was characterized by the presence of low mood, a sense of social detachment, self-incrimination, feelings of guilt, tears, loss of appetite, indecisiveness, increased fatigue, difficulties at work.

The dysphoric variant in the clinical structure had a combination of a steady decrease in mood with irritability, conflict,

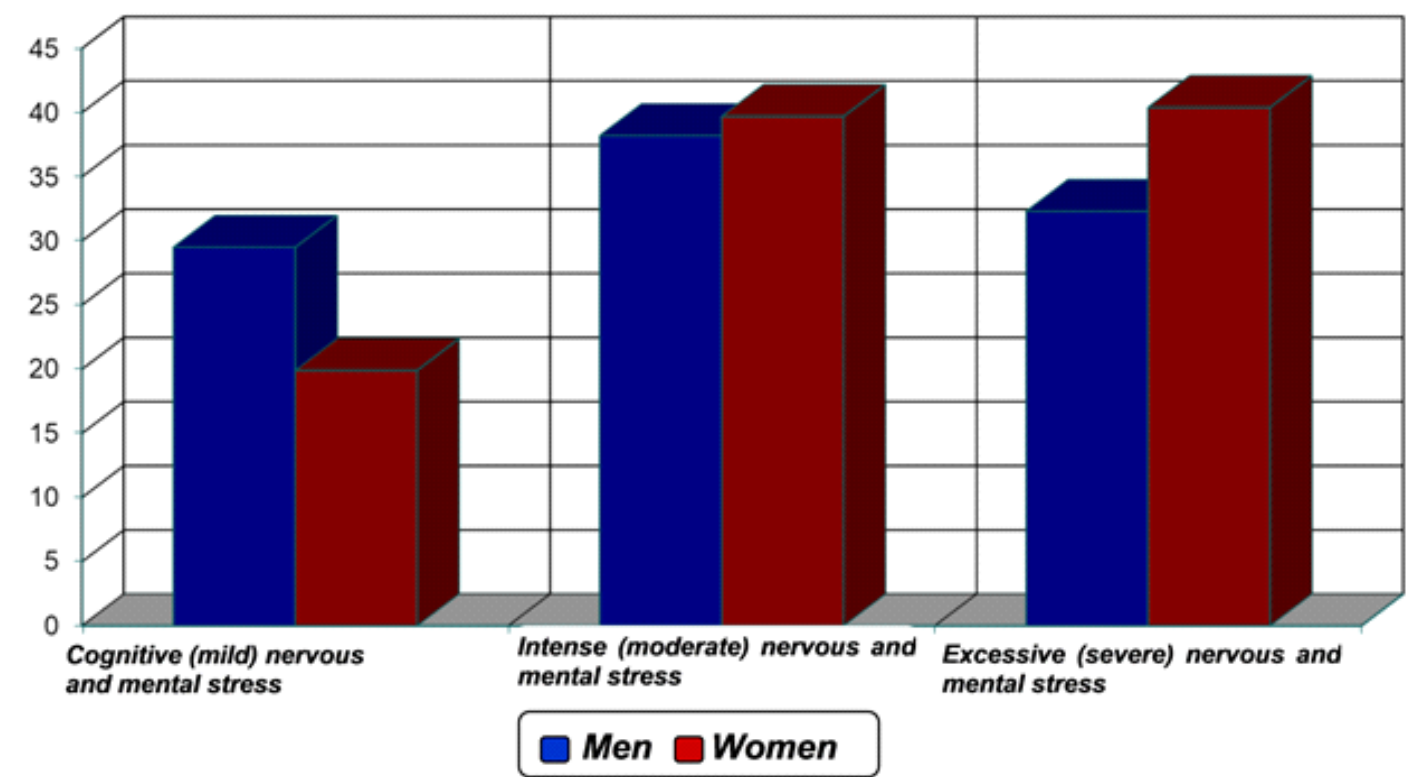

Fig. 5. Level of expressiveness of nervous and mental stress in patients with mitochondrial pathology $(\mathrm{p}<0.05 ; \mathrm{p} \leq 0.001)$

dysphoric and depressive-apatitic ( $15.6 \%$ each) variants. Fig. 6.

In half of the patients $(50.3 \%)$ an anxiodepressive variant of the depressive disorder was recorded, which was manifested by the presence of low mood secondary to anxiety, dissatisfaction with oneself, fears without real causes, sleep disturbances, self-excuse, and feeling of lucidity. dissatisfaction, suicidal thoughts, loss of body weight, sleep disturbance.

The depressive-apathetic variant was characterized by reduced activity on the background of emotional oppression, a sense of agony, loss of sexual desire, the patient had difficulties performing simple self-service functions, they spent a lot of time in bed, were not interested in others, did not feel hunger, refused to eat.

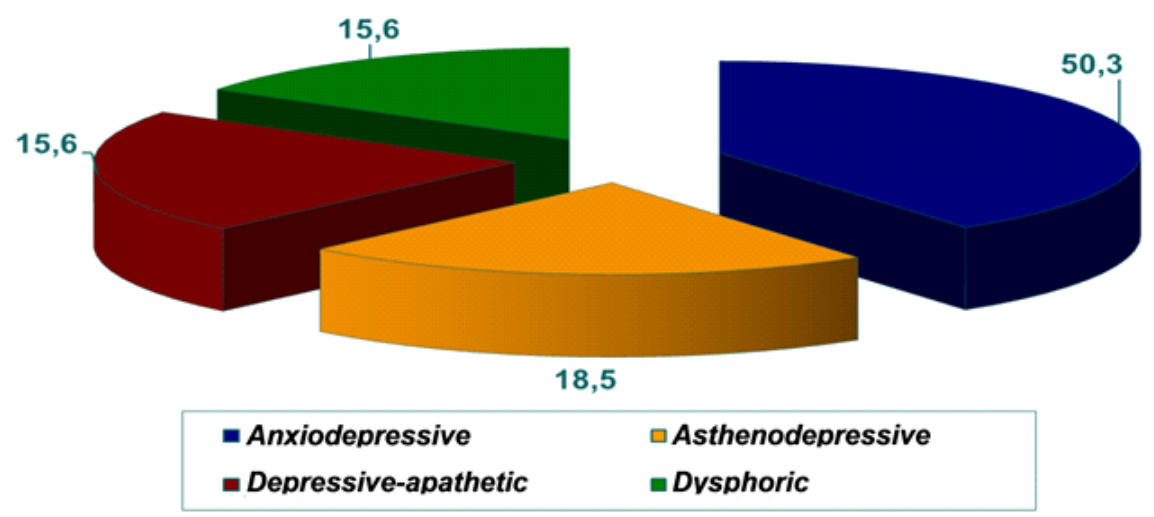

Fig. 6. Variants of depressive disorders in patients with mitochondrial pathology $(\% ; p<0.05)$ 
At the next stage of the study, a screening survey of 14 families of patients with mitochondrial pathology, accompanied by depressive disorders and 12 families of patients with mitochondrial pathology without affective disorders was conducted.

Among the issues, there were those relating to the health of parents of patients and the closest relatives (along the lines of father and mother).

When processing the results, the following data was obtained:

- In the first group of patients with relatives on the mother's side, in contrast to the parent, a higher frequency of psychosomatic symptoms and depression was detected $(59.1 \%$ and $48.3 \%$ respectively).

- In the second group, $15.7 \%$ and $10.3 \%$ of the respondents met psychosomatic and depressive symptoms.

- On the father's line in both groups, these figures were $7.4 \%$ and $9.2 \%$ respectively.

On the basis of the obtained data, a comprehensive system of correction and prevention of depression was developed in patients with mitochondrial pathology, which included pharmacotherapy, psychotherapy and psychoeducation.

Patients with mild manifestations of depression provided psychotherapeutic assistance in the form of cognitive-behavioral, family psychotherapy, cognitive training, training for solving interpersonal problems.

Patients with moderate and severe manifestations of depression received both psychotherapeutic care and therapy with antidepressants. Preference was given to selective serotonin reuptake inhibitors (SSRI) at doses corresponding to the severity of depressive disorders.

With all patients, regardless of the severity of depression, psychoeducational work was conducted. The patients' families were also accepted with psycho-educational assistance. Psychoeducation was aimed at various aspects of the disease: biological, psychological and social. Patients explained the purpose of treatment, how to achieve a positive outcome of treatment, the advantages and disadvantages of different therapies. With each patient individually and in group, a crisis plan was worked out, that is what to do in the case of recurrence, whom to address, what drugs to take, etc.

Before the start of therapy, as well as in the second, fourth and sixth weeks of treatment, the dynamics of changes in the state of depression on the Hamilton scale was estimated in patients.

The obtained results are presented in Fig. 7.

According to the results of the complex system of treatment, patients managed to achieve reduction of anxiodepressive disorders $(73.5 \%$ of patients), decrease in motor and ideatric inhibition $(65.2 \%)$, decrease in the duration and intensity of depressive appetite $(72.5 \%)$, recovery of interest to activities that previously interested patients $(69.5 \%)$, improvement of psychosocial adaptation $(81.3 \%)$, transition of maladaptive types of attitude to the disease into adaptive $(66.5 \%)$, improvement of the quality of life of patients $(83.2 \%)$.

\section{Dynamics of Hamilton Scale indicators under the influence of integrated therapy}
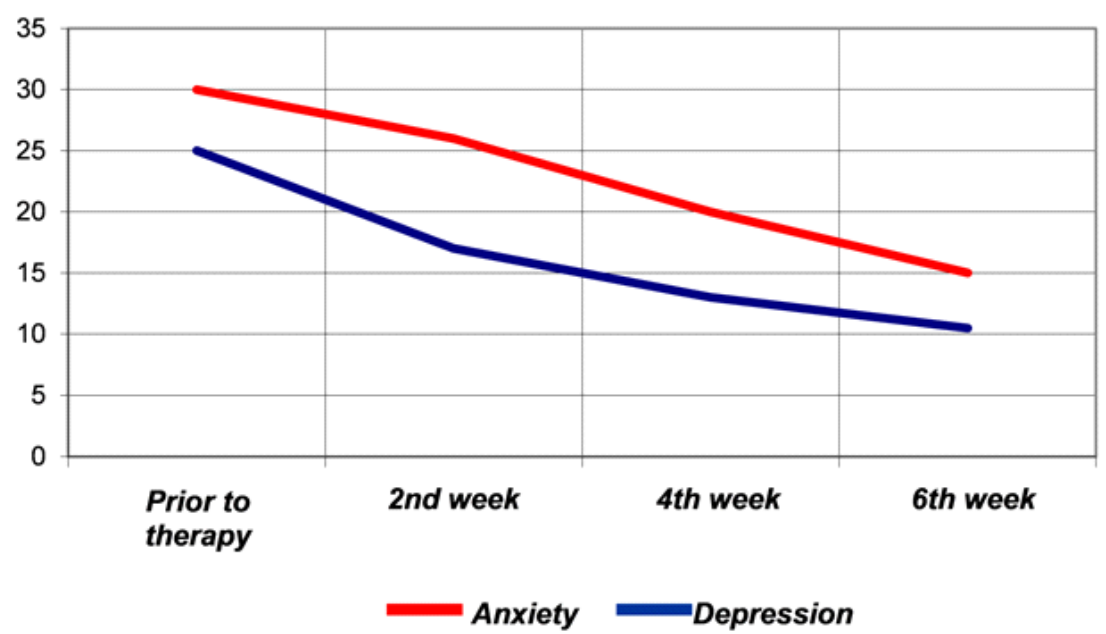

Fig. 7. Dynamics of changes in the state of depression on the Hamilton scale $(p<0.05)$ 
Over the past 12 months, foreign scientists Burnet B. and co-authors (2005) surveyed patients with mitochondrial diseases and their family members, especially maternal relatives. The results obtained in their work indicate a higher frequency of migraines in combination with irritable bowel syndrome and depression. At the time, our study, which lasted for 3.5 years, not only revealed the presence of depressive disorders in the surveyed contingent, but also identified different variants of depression manifestations. In our work, in maternal relatives of patients, unlike paternal relatives, it was found a higher frequency of psychosomatic symptoms and depression, in contrast to the study by Burnet B. and co-authors [19]. In the study of Babenko V.N. and co-authors (2018), an experiment with laboratory animals (male mice) confirmed that persistent social conflicts cause severe mitochondrial dysfunction in the brain [18]. In our study, patients and their family members who had internal family problems and impaired social functioning were more likely to have clinical manifestations of depressive disorders. Researchers Kato T.M., Kubota-Sakashita M., Fujimory-Tonou N. et al. (2018) have shown that mitochondrial dysfunction influences the activity of serotonin neurons in mice with a mutation of the ANT1 gene and is a predictor of the emergence of neuron-mediated depressive-like episodes [20]. It can be assumed that the presence of such a mutant gene in a person with mitochondrial pathology may be a signal indicator of the emergence of depressive disorders and will allow to extract it as a diagnostic marker. These findings are of scientific interest and are promising for further studies.

The results obtained during the study do not contradict the previous foreign studies, but do have some differences in the number of patients, their age and the duration of the study. In the course of work the variants of depressive disorders were distinguished, certain differences in manifestations of depressive disorders between women and men with mitochondrial pathology were revealed.

Analyzing the information received regarding the approaches to correction of mental disorders in the mitochondrial pathology, it can also be noted that antidepressants can also affect brain metabolism and mitochondrial activity. This effect has differences in different parts of the brain and is regulatory.

\section{Conclusions}

Consequently, the several stages of the study made it possible to distinguish the most common clinical and psychopathological features of the examined patients with mitochondrial pathology, which was complicated by depressive disorders. In patients with mitochondrial pathology with signs of depression, moderate levels of depression and intense (moderate) neuro-mental stress dominated. The structure of depressive disorders was dominated by anxiety-depressive symptom complex with a low mood secondary to anxiety, dissatisfaction with oneself, fears without real causes, sleep disturbances, self-prosecution, and feeling of lucidity. In the families of patients with mitochondrial pathology, accompanied by depressive symptoms, relatives on the mother's side, in contrast to the father's side, were found to have a higher incidence of psychosomatic symptoms and depression.

The time course of a depressed state was evaluated during therapy and psychotherapeutic interventions, psychoeducation and was characterized by positive changes.

Pharmacotherapy and psychotherapy were differentiated and depended on clinically psychopathological manifestations of depressive disorders, their severity and general condition of patients. The complex of psychotherapeutic methods included cognitive-behavioral, family psychotherapy, cognitive training and training to solve interpersonal problems. Psychoeducational assistance was provided to the patients and their families.

\section{References}

1. Sukhorukov V.S. (2008). Mitochondrialnya patologia i problemy patogenesa psyhicheskih narushenii. Zhurnal nevrologii i psychiatrii im. Korsakova S.S. 108(6), 83-90.

2. Sukhorukov V.S. (2011). Ocherki mitochondrialnoy patologii. Moskov, Medpractika, 288.

3. Gardner A., Boles R.G. (2005). Is a "Mitochondrial Psychiatry" in the Future? A Review. Current Psychiat Rev., 1(3), 255-271.

4. Gardner A., Pagani M., Wibom R. et al. (2003). Alterations of rcbf and mitochondrial dysfunction in major depressive disorder: a case report. Act a Psychiat. Scand. https: doi.org 10.1034 j.16000447.2003.02188.x. 
5. Gardner A. (2004). Mitochondrial dysfunction and alterations of brain HMPAO SPECT in depressive disorder - perspectives on origins of "somatization" [doctoral thesis]. Karolinska Institutet, Neurotec Institution, Division of Psychiatry, Stockholm, 234.

6. Konradi C., Eaton M., MacDonald M. L. et al. (2004). Molecular evidence for mitochondrial dysfunction in bipolar disorder. Arch Gen Psychiatry, 61, 300-308.

7. Marchbanks R. M., Ryan M., Day I. N. et al. (2003). A mitochondrial DNA sequence variant associated with schizophrenia and oxidative stress. Schizophrenia Res., 65, 33-38.

8. Munakata K., Tanaka M., Mori K. et al. (2004). Mitochondrial DNA 3644T $>$ C mutation associated with bipolar disorder. Genomics, 84, 1041-1050.

9. Newman-Toker D. E., Horton J. C., Lessell S. (2003). Recurrent visual loss in Leber hereditary optic neuropathy. Arch Ophthalmol, 121, 288-291.

10.Nesbitt V., Pitceathly R.D., Turnbull D.M. et al. (2013). The UK MRC Mitochondrial Disease Patient Cohort Study: clinical phenotypes associated with the m.3243A $>$ G mutation-implications for diagnosis and management. J Neurol Neurosurg Psychiatry, 84(8), 936-8. https: doi: 10.1136 jnnp2012-303528.

11. Odawara M. (2002). Mitochondrial gene abnormalities as a cause of psychiatric diseases. Nucleic Acids Res, 2, 253-254.

12.Payne B.A., Wilson I.J., Yu-Wai-Man P. et al. (2013). Universal heteroplasmy of human mitochondrial DNA. Human Molecular Genetics, 22 (2), 384-390.

13. Rango M., Bozzali M., Prelle A. et al. (2001). Brain activation in normal subjects and in patients affected by mitochondrial disease without clinical central nervous system involvement: a phosphorus magnetic resonance spectroscopy study. J Cereb Blood Flow Metab., 21, 85-91.

14. Wang Q., Ito M., Adams K. et al. (2004). Mitochondrial DNA control region sequence variation in migraine headache and cyclic vomiting syndrome. Am J Med Genet, 131A, 50-58.

15. Washizuka S., Kakiuchi C., Mori K. et al. (2003). Association of mitochondrial complex I subunit gene NDUFV2 at 18p11 with bi polar disorder. Am J Med Genet, 120B, 72-78.

16. Hamilton, M (1967). Development of a rating scale for primary depressive illness. British Journal of Social and Clinical Psychology 6: 278-96 PMID.

17. Nemchin, T.A. (1983). Sostoyaniya nervno-psirhicheskogo napryazheniya. States of mental stress. Leningrad, 167.

18. Babenko, V.N., Smagin, D.A., Galyamina, A.G. et al. (2018). Altered Slc25 family gene expression as markers of mitochondrial dysfunction in brain regions under experimental mixed anxiety/depressionlike disorder. BMC Neurosci 19, 79. https: doi.org 10.1186 S 12868-018-0480-6.

19.Burnet B.B., Gardner A., Boles R.G. Mitochondrial inheritance in depression, dysmotility and migraine? J Affect Disord. 2005; 88: 109-116.

20.Kato T. M., Kubota-Sakashita M., Fujimory-Tonou N. et al. (2018). Ant1 mutant mice bridge the mitochondrial and serotonergic dysfunctions in bipolar disorder. Molecular Psychiatry, 23, 2039 - 2049.

Received: 03-Jun-2019

Accepted: 02-Mar-2020 